\title{
Stochastic stability analysis for a neutral-type neural networks with Markovian jumping parameters
}

\author{
Song Guo, Bo Du* \\ Department of Mathematics, Huaiyin Normal University, Huaian Jiangsu, 223300, P. R. China. \\ Communicated by R. Saadati
}

\begin{abstract}
In this paper, the stability problem is studied for a class of stochastic neutral-type neural networks with Markovian jumping parameters. By using fixed point theorem, the existence and uniqueness of solution for the neural networks system are obtained. Furthermore, based on the Lyapunov-Krasovskii functional, a linear matrix inequality (LMI) approach is developed to establish sufficient conditions to guarantee the mean square stability of the neural networks. An example is given to show the effectiveness of the proposed stability criterion. (C)2017 All rights reserved.
\end{abstract}

Keywords: Markovian jumping parameters, linear matrix inequality, mean square stability. 2010 MSC: 34B15.

\section{Introduction}

In the past decades, neural networks have received considerable attention due to a variety of applications, such as system recognition, signal processing, static image processing, target tracking and associative memory $[1,6,15,16]$. Particularly, the work on time-delay neural networks has received great attention due to time-delay is an inherent feature of many processes, such as physical and chemical processes, biological systems and nuclear reactors may lead to instability or poor performances for the corresponding closed-loop systems, see, e.g., $[3,9,10,18]$. Recently, many results have been obtained for neutral-type neural networks. For example, Liu et al. [7] concerned a class of Markovian jumping neutral-type neural networks with mode-dependent mixed time-delays:

$$
\begin{aligned}
\dot{x}(t)= & E(r(t)) \dot{x}\left(t-\tau_{1, r(t)}\right)-A(r(t)) x(t)-B(r(t)) f(x(t)) \\
& +c(r(t)) g\left(x\left(t-\tau_{2, r(t)}\right)\right)+D(r(t)) \int_{t-\tau_{3, r(t)}}^{t-\tau_{4, r(t)}} h(x(s)) d s .
\end{aligned}
$$

The authors $([5,12])$ investigated the following neutral-type neural systems:

$$
\left\{\begin{array}{l}
\left(x_{i}\right)^{\prime}(t)=-a_{i}(t) x_{i}(t)+\sum_{j=1}^{n}\left[b_{i j}(t) f_{j}\left(t, x_{j}(t)\right)+d_{i j}(t) g_{j}\left(t, x_{j}^{\prime}\left(t-\tau_{i j}(t)\right)\right)\right]+I_{i}(t), \\
x_{i}(t)=\phi_{i}(t), t \in[-\tau, 0], i=1,2, \cdots, n,
\end{array}\right.
$$

\footnotetext{
*Corresponding author

Email addresses: guosong77@hytc.edu.cn (Song Guo), dubo7307@163.com (Bo Du)
} 
and

$$
\begin{aligned}
y^{\prime}(t) & =-A y(t)+B g(y(t))+C g(y(t-\tau(t)))+D y^{\prime}(t-h(t)), \quad t \neq t_{k}, \\
\Delta y(t) & =I_{k}(y(t)), \quad t=t_{k}, \\
y\left(t_{0}^{+}+s\right) & =\phi(s), \quad s \in\left[t_{0}-\rho, t_{0}\right], k \in \mathbb{N} .
\end{aligned}
$$

More detailed results on neural networks could be found in [11,14,17] and the references therein.

So far, to the best of the authors' knowledge, there is few results for the stability problems to neutraltype neural networks with Markovian parameters and mixed time delays. The major challenge lists as follows: (1) in order to construct a feasible Lyapunov-Krasovskii functional, the properties of neutral operator $\mathcal{A}$ (defined in (2.2)) can be considered. So, studying the properties of the operator $\mathcal{A}$ seems very important; (2) when the non-constant delays exist in neural networks, the corresponding stability problems become more complicated since a new Lyapunov functional is required to reflect variable delay's influence; and (3) it is non-trivial to establish a unified framework to handle the Markovian parameters, neutral terms and variable delays influence. It is, therefore, the main purpose of this paper to make the first attempt to handle the listed challenges.

In this paper, we consider the stability problems for a neutral-type neural networks with Markovian parameters and mixed delays. Note that neural system includes Markovian parameters, the neutral term and variable delays that are all dependent on the properties of neutral operator. The purpose of this paper is to obtain some existence, asymptotically stable and exponential stable results. A numerically efficient LMI approach is developed to solve the addressed problems. A simulation example is used to demonstrate the usefulness of the LMI method. The contribution of this paper is threefold. (1) For obtaining the existence results, the neutral operator is first taken into account in the neural networks with Markovian parameters and mixed time delays and a non-neutral system can be viewed as the special cases. (2) Different from most of the existing results, we develop a new unified framework to cope with the stability problems for the neural networks by a blend of matrix theory, Lyapunov-Krasovskii functional and LMI approach, which may be of independent interest. It is worth pointing out that our main results are also valid for the case of non-neutral system. (3) Some new techniques are used in this paper. In particular, a key inequality and an appropriate Lyapunov-Krasovskii functional will be introduced to handle the neural networks, and they play a crucial role in the derivation of our main results.

Throughout the manuscript, $\mathbb{R}^{n}$ and $\mathbb{R}^{n \times m}$ denote, respectively, the $n$-dimensional Euclidean space and the set of all $n \times m$ real matrices. The superscript "T" denotes the matrix transposition. We will use the notation $A>0$ (or $A<0$ ) to denote that $A$ is a symmetric and positive definite (or negative definite) matrix. If $A, B$ are symmetric matrices, $A>B(A \geqslant B)$, then $A-B$ is a positive definite (positive semi-definite). $|z|$ denotes the Euclidean norm of a vector $z$ and $\|A\|$ denotes the induced norm of the matrix $A$, that is $\|A\|=\sqrt{\lambda_{\max }\left(A^{\top} A\right)}$ where $\lambda_{\max }(\cdot)$ means the largest eigenvalue of $A$. $\mathbb{E}[x]$ and $\mathbb{E}[x \mid y]$ mean the expectation of $x$ and the expectation of $x$ conditional on $y$, respectively. If their dimensions are not explicitly stated, are assumed to be compatible for algebraic operations.

The following sections are organized as follows. In Section 2, we give problem formulation, some useful lemmas, and definitions. In Section 3, sufficient conditions are established for existence results of system (2.3). The main results of the present paper are given in Section 4. In Section 5, a numerical example is given to show the feasibility of our results. Finally, some conclusions are given about this paper.

\section{Problem formulation}

Let $r(t)(t \geqslant 0)$ be a right-continuous Markovian chain taking values in a finite sate space $S=$ $\left\{1,2, \cdots, n_{0}\right\}$ with generator $\Pi=\left\{\pi_{i j}\right\}$ given by

$$
P\{r(t+\Delta)=j \mid r(t)=i\}= \begin{cases}\pi_{i j} \Delta+o(\Delta), & \text { if } i \neq j, \\ 1+\pi_{i j} \Delta+o(\Delta), & \text { if } \mathfrak{i}=j .\end{cases}
$$


Here $\Delta>0$ and $\pi_{i j} \geqslant 0$ is the transition rate from $i$ to $j$ if $i \neq j$ and $\pi_{i i}=-\sum_{j \neq i} \pi_{i i}$.

Consider the following neutral-type neural networks with Markovian jumping parameters:

$$
\left(\mathcal{A}_{p} x_{p}\right)^{\prime}(t)=-c_{p}(r(t)) x_{p}(t)+\sum_{q=1}^{n} a_{p q}(r(t)) f_{q}\left(x_{q}(t)\right)+\sum_{q=1}^{n} b_{p q}(r(t)) g_{q}\left(x_{q}(t-\tau(r(t)))\right),
$$

where $p=1,2, \cdots, n,\left(\mathcal{A}_{p} x_{p}\right)(t)=x_{p}(t)-d_{p} x_{p}(t-\gamma(r(t))), x_{p}(t)$ represents the state of the ith neuron at time $t, f_{p}$ and $g_{p}$ are the activation functions of the pth neuron, $c_{p}$ represents the rate with which the $p$ th unit will reset its potential to the resting state when disconnected from the network and external inputs, $a_{p q}$ denotes the strength of the qth unit on the $p$ th unit, $b_{p q}$ denotes the strength of the qth unit on the pth unit, and $\tau(r(t))$ and $\gamma(r(t))$ denote the discrete delays of networks in the mode $r(t)$. Denote

$$
\begin{aligned}
\mathcal{A x}(\mathrm{t}) & =x(\mathrm{t})-\mathrm{D} x(\mathrm{t}-\gamma(\mathrm{r}(\mathrm{t}))), \mathrm{D}=\operatorname{diag}\left(\mathrm{d}_{1}, \mathrm{~d}_{2}, \cdots, \mathrm{d}_{\mathrm{n}}\right), \\
\mathcal{A x}(\mathrm{t}) & =\left(\mathcal{A}_{1} x_{1}(\mathrm{t}), \mathcal{A}_{2} \mathrm{x}_{2}(\mathrm{t}), \cdots, \mathcal{A}_{\mathrm{n}} \mathrm{x}_{\mathrm{n}}(\mathrm{t})\right)^{\top}, \\
\mathrm{C} & =\operatorname{diag}\left(\mathrm{c}_{1}(\mathrm{r}(\mathrm{t})), \mathrm{c}_{2}(\mathrm{r}(\mathrm{t})), \cdots, \mathrm{c}_{\mathrm{n}}(\mathrm{r}(\mathrm{t}))\right), \\
\mathrm{A} & =\left[\mathrm{a}_{\mathrm{pq}}(\mathrm{r}(\mathrm{t}))\right]_{\mathrm{n} \times \mathrm{n}}, \mathrm{B}=\left[\mathrm{b}_{\mathrm{pq}}(\mathrm{r}(\mathrm{t}))\right]_{\mathrm{n} \times \mathrm{n}}, \\
\mathrm{F}(\mathrm{x}(\mathrm{t})) & =\left(\mathrm{f}_{1}\left(\mathrm{x}_{1}(\mathrm{t})\right), \mathrm{f}_{2}\left(\mathrm{x}_{2}(\mathrm{t})\right), \cdots, \mathrm{f}_{\mathrm{n}}\left(\mathrm{x}_{\mathrm{n}}(\mathrm{t})\right)\right)^{\top}, \\
\mathrm{G}(\mathrm{x}(\mathrm{t}-\tau(\mathrm{r}(\mathrm{t})))) & =\left(\mathrm{g}_{1}\left(\mathrm{x}_{1}(\mathrm{t}-\tau(\mathrm{r}(\mathrm{t})))\right), \mathrm{g}_{2}\left(\mathrm{x}_{2}(\mathrm{t}-\tau(\mathrm{r}(\mathrm{t})))\right), \cdots, \mathrm{g}_{\mathrm{n}}\left(\mathrm{x}_{\mathrm{n}}(\mathrm{t}-\tau(\mathrm{r}(\mathrm{t})))\right)\right)^{\top} .
\end{aligned}
$$

Then system (2.1) can be rewritten as

$$
(\mathcal{A x})^{\prime}(\mathrm{t})=-\mathrm{C}(\mathrm{r}(\mathrm{t})) x(\mathrm{t})+\mathrm{A}(\mathrm{r}(\mathrm{t})) \mathrm{F}(\mathrm{x}(\mathrm{t}))+\mathrm{B}(\mathrm{r}(\mathrm{t})) \mathrm{G}(\mathrm{x}(\mathrm{t}-\tau(\mathrm{r}(\mathrm{t})))) .
$$

Remark 2.1. The neural network (2.3) shows the neutral character by the operator $\mathcal{A}$, which is different from other papers, see, e.g., $[11,12,17]$.

Remark 2.2. When $\mathrm{D}(\mathrm{r}(\mathrm{t}))=0, \mathrm{r}(\mathrm{t}) \in \mathrm{S}$, system (2.3) is changed into a non-neutral-type discrete neural networks which has been extensively studied, see, e.g., [1, 3, 5, 9, 10, 14, 16, 18].

For activation functions, we need the following assumptions.

Assumption 2.3. For $p \in\{1,2, \cdots, n\}$, the neuron activation functions in (2.1) satisfy

$$
l_{p}^{-} \leqslant \frac{f_{p}\left(s_{1}\right)-f_{p}\left(s_{2}\right)}{s_{1}-s_{2}} \leqslant l_{p}^{+}, \quad \sigma_{p}^{-} \leqslant \frac{g_{p}\left(s_{1}\right)-g_{i}\left(s_{2}\right)}{s_{1}-s_{2}} \leqslant \sigma_{p}^{+},
$$

where $l_{p}^{-}, l_{p}^{+}$and $\sigma_{p}^{-}, \sigma_{p}^{+}$are some constants.

Lemma 2.4. If $\mathrm{d}_{\mathrm{p}}^{+}<1$, then the inverse of difference operator $\mathcal{A}$ denoted by $\mathcal{A}^{-1}$, exists and

$$
\frac{1}{1-\mathrm{d}_{\mathrm{p}}^{-}} \leqslant\left|\mathcal{A}^{-1}\right| \leqslant \frac{1}{1-\mathrm{d}_{\mathrm{p}}^{+}}
$$

where $d_{p}^{+}=\max \left\{\left|d_{1}\right|,\left|d_{2}\right|, \cdots,\left|d_{n}\right|\right\}, d_{p}^{-}=\min \left\{\left|d_{1}\right|,\left|d_{2}\right|, \cdots,\left|d_{n}\right|\right\}$.

Proof. Let $\mathrm{B} x(\mathrm{t})=\mathrm{Dx}(\mathrm{t}-\gamma(\mathrm{r}(\mathrm{t})))$, then $|\mathrm{B}|=\mathrm{d}_{\mathrm{p}}^{+}<1$. Thus, $\mathcal{A}^{-1}=(\mathrm{I}-\mathrm{B})^{-1}$ exists and $\left|\mathcal{A}^{-1}\right|=\mid(\mathrm{I}-$ $\mathrm{B})^{-1} \mid \leqslant \frac{1}{1-\mathrm{d}_{\mathrm{p}}^{+}}$. Obviously, $\left|\mathcal{A}^{-1}\right| \geqslant \frac{1}{1-\mathrm{d}_{\mathrm{p}}^{-}}$.

Lemma 2.5 ([2]). Given constant matrices $\Omega_{1}, \Omega_{2}, \Omega_{3}$ where $\Omega_{1}=\Omega_{1}^{\top}$ and $\Omega_{2}>0$, then

$$
\Omega_{1}+\Omega_{3}^{\top} \Omega_{2}^{-1} \Omega_{3}<0
$$

if only if

$$
\left(\begin{array}{cc}
\Omega_{1} & \Omega_{3}^{\top} \\
\Omega_{3} & -\Omega_{2}
\end{array}\right)<0 \quad \text { or } \quad\left(\begin{array}{cc}
-\Omega_{2} & \Omega_{3} \\
\Omega_{3}^{\top} & -\Omega_{1}
\end{array}\right)<0
$$


Lemma 2.6 ([8]). Suppose that $B=\operatorname{diag}\left\{\beta_{1}, \beta_{2}, \cdots, \beta_{n}\right\}$ is a positive semi-definite matrix. Let

$$
x(t)=\left(x_{1}, x_{2}, \cdots, x_{n}\right)^{\top} \in \mathbb{R}^{n}
$$

and $\mathrm{H}(\mathrm{t})=\left(\mathrm{h}_{1}, \mathrm{~h}_{2}, \cdots, \mathrm{h}_{\mathrm{n}}\right)^{\top}$ be a continuous nonlinear function satisfying

$$
l_{i}^{-} \leqslant \frac{h_{i}(s)}{s} \leqslant l_{i}^{+}, s \neq 0, s \in \mathbb{R}, i=1,2, \cdots, n
$$

with $l_{i}^{-}$and $l_{i}^{+}$being constant scalars. Then

$$
\left(\begin{array}{c}
x \\
H(x)
\end{array}\right)^{\top}\left(\begin{array}{cc}
\mathrm{BL}_{1} & -\mathrm{BL}_{2} \\
-\mathrm{BL}_{2} & \mathrm{~B}
\end{array}\right)\left(\begin{array}{c}
x \\
\mathrm{H}(\mathrm{x})
\end{array}\right) \leqslant 0, \quad \text { or } \quad x^{\top} \mathrm{BL}_{1} x-2 x^{\top} \mathrm{B} \mathrm{L}_{2} \mathrm{H}(\mathrm{x})+\mathrm{H}^{\top}(x) \mathrm{BH}(\mathrm{x}) \leqslant 0,
$$

where

$$
\mathrm{L}_{1}=\operatorname{diag}\left\{\mathrm{l}_{1}^{+} \mathrm{l}_{1}^{-}, \mathrm{l}_{2}^{+} \mathrm{l}_{2}^{-}, \cdots, \mathrm{l}_{\mathrm{n}}^{+} \mathrm{l}_{\mathrm{n}}^{-}\right\} \quad \text { and } \quad \mathrm{L}_{2}=\operatorname{diag}\left\{\left(\mathrm{l}_{1}^{+}+\mathrm{l}_{1}^{-}\right) / 2,\left(\mathrm{l}_{2}^{+}+\mathrm{l}_{2}^{-}\right) / 2, \cdots,\left(\mathrm{l}_{\mathrm{n}}^{+}+\mathrm{l}_{\mathrm{n}}^{-}\right) / 2\right\} .
$$

Lemma 2.7 ([4]). For any positive definite matrix $M>0$, scalar $\gamma>0$, vector function $\omega:[0, \gamma] \rightarrow \mathbb{R}^{n}$ such that the integrations concerned are well-defined, the following inequality holds:

$$
\left(\int_{0}^{\gamma} \omega(s) d s\right)^{\top} M\left(\int_{0}^{\gamma} \omega(s) d s\right) \leqslant \gamma\left(\int_{0}^{\gamma} \omega^{\top}(s) M \omega(s) d s\right)
$$

Definition 2.8. The neural network (2.3) is said to be asymptotically stable in mean square if, for any solution $x(t)$ of $(2.3)$, the following holds:

$$
\lim _{t \rightarrow \infty} \mathbb{E}\left[|x(t)|^{2}\right]=0 .
$$

Furthermore, the network (2.3) is said to be exponentially stable in mean square if, for any solution $x(t)$ of (2.3), there exist constants $\alpha>0$ and $\mu>0$ such that

$$
\mathbb{E}\left[|x(t)|^{2}\right] \leqslant \mu e^{-\alpha t} .
$$

We need the following denotations:

$$
\begin{aligned}
\bar{\tau} & =\max \{\tau(i), i \in S\}, & \underline{\tau} & =\min \{\tau(i), i \in S\}, \quad \bar{\pi}=\max \left\{\pi_{i i}, i \in S\right\}, \\
v_{1} & =\operatorname{diag}\left\{l_{1}^{+} l_{1}^{-}, l_{2}^{+} l_{2}^{-}, \ldots, l_{n}^{+} l_{n}^{-}\right\}, & v_{2} & =\operatorname{diag}\left\{\left(l_{1}^{+}+l_{1}^{-}\right) / 2,\left(l_{2}^{+}+l_{2}^{-}\right) / 2, \cdots,\left(l_{n}^{+}+l_{n}^{-}\right) / 2\right\}, \\
\omega_{1} & =\operatorname{diag}\left\{\sigma_{1}^{+} \sigma_{1}^{-}, \sigma_{2}^{+} \sigma_{2}^{-}, \cdots, \sigma_{n}^{+} \sigma_{n}^{-}\right\}, & \omega_{2} & =\operatorname{diag}\left\{\left(\sigma_{1}^{+}+\sigma_{1}^{-}\right) / 2,\left(\sigma_{2}^{+}+\sigma_{2}^{-}\right) / 2, \cdots,\left(\sigma_{n}^{+}+\sigma_{n}^{-}\right) / 2\right\} .
\end{aligned}
$$

\section{Existence of solution of system (2.3)}

Theorem 3.1. If $\mathrm{d}_{\mathrm{p}}^{+}<1(\mathrm{p}=1,2, \cdots, \mathrm{n})$ and Assumption 2.3 hold, furthermore the following condition satisfies:

$$
\frac{h}{1-d_{p}^{+}}\|A\|+\frac{h\left(\hat{l}_{p}\right)^{+}}{1-d_{p}^{+}}\|B\|+\frac{h\left(\hat{\sigma}_{p}\right)^{+}}{1-d_{p}^{+}}<1
$$

where

$$
\begin{aligned}
\hat{l}_{p} & =\max _{p \in\{1,2, \cdots, n\}}\left\{\left|l_{p}^{+}\right|,\left|l_{p}^{-}\right|\right\}, & \hat{\sigma}_{p} & =\max _{p \in\{1,2, \cdots, n\}}\left\{\left|\sigma_{p}^{+}\right|,\left|\sigma_{p}^{-}\right|\right\}, \\
\left(\hat{l}_{p}\right)^{+} & =\max \left\{\hat{l}_{p}\right\}, & \left(\hat{\sigma}_{p}\right)^{+} & =\max \left\{\hat{\sigma}_{p}\right\}, p \in\{1,2, \cdots, n\} .
\end{aligned}
$$

Then solution of system (2.3) exists in $\mathcal{B}=\mathrm{C}\left([0, \mathrm{~h}], \mathbb{R}^{\mathrm{n}}\right)$ and is unique which satisfies initial condition $\mathrm{x}=$ $\phi(s), s \in[-\tau, 0]$, where $h$ and $\tau$ are positive constants. 
Proof. Let $x \in \mathcal{B}$ and $\mathcal{A x}(\mathrm{t})=\mathrm{u}(\mathrm{t})$, then $x(\mathrm{t})=\mathcal{A}^{-1} \mathrm{u}(\mathrm{t})$. Let $\mathrm{r}(\mathrm{t})=i, i \in S$, (2.3) transforms to the following system:

$$
\mathfrak{u}^{\prime}(\mathrm{t})=-\mathrm{C}(\mathfrak{i})\left[\mathcal{A}^{-1} \mathfrak{u}(\mathrm{t})\right]+\mathrm{A}(\mathfrak{i}) \mathrm{F}\left(\left[\mathcal{A}^{-1} \mathfrak{u}(\mathrm{t})\right]\right)+\mathrm{B}(\mathfrak{i}) \mathrm{G}\left(\mathcal{A}^{-1} \mathfrak{u}(\mathbf{t}-\tau(\mathfrak{i}))\right) .
$$

Define $T$ on $\mathcal{B}$ by

$$
\mathrm{Tu}(\mathrm{t})=\mathrm{u}(0)+\int_{0}^{\mathrm{t}}\left\{-\mathrm{A}\left[\mathcal{A}^{-1} \mathfrak{u}(\mathrm{s})\right]+\mathrm{BF}\left(\left[\mathcal{A}^{-1} \mathbf{u}(\mathrm{s})\right]\right)+\mathrm{DG}\left(\mathrm{A}^{-1} \mathrm{u}(\mathrm{s}-\tau(\mathfrak{i}))\right)\right\} \mathrm{ds} .
$$

Obviously, $\mathrm{Tu} \in \mathcal{B}$. We will show that $\mathrm{T}: \mathcal{B} \rightarrow \mathcal{B}$ is a contraction mapping. In fact, from Lemma 2.4 and Assumption 2.3, for any $u, v \in \mathcal{B}$ we have

$$
\begin{aligned}
|\mathrm{Tu}-\mathrm{T} v| \leqslant & \int_{0}^{t}\left\{\left|A\left[\mathcal{A}^{-1} \mathrm{u}(\mathrm{s})-\mathcal{A}^{-1} v(s)\right]\right|+\left|\mathrm{B}\left[\mathrm{F}\left(\left[\mathcal{A}^{-1} \mathrm{u}(\mathrm{s})\right]\right)-\mathrm{F}\left(\left[\mathcal{A}^{-1} v(s)\right]\right)\right]\right|\right. \\
& +\left|\mathrm{D}\left[\mathrm{G}\left(\mathrm{A}^{-1} \mathrm{u}(\mathrm{s}-\tau(\mathfrak{i}))\right)-\mathrm{G}\left(\mathrm{A}^{-1} \mathcal{v}(\mathrm{s}-\tau(\mathfrak{i}))\right)\right]\right| \\
\leqslant & \left(\frac{\mathrm{h}}{1-\mathrm{d}_{\mathrm{p}}^{+}}\|\mathrm{A}\|+\frac{\mathrm{h}\left(\hat{\mathrm{l}}_{\mathrm{p}}\right)^{+}}{1-\mathrm{d}_{\mathrm{p}}^{+}}\|\mathrm{B}\|+\frac{\mathrm{h}\left(\hat{\sigma}_{\mathrm{p}}\right)^{+}}{1-\mathrm{d}_{\mathrm{p}}^{+}}\right)|\mathrm{u}-\boldsymbol{v}|
\end{aligned}
$$

The condition $\frac{h}{1-d_{p}^{+}}\|A\|+\frac{h\left(\hat{\mathfrak{l}}_{p}\right)^{+}}{1-d_{\mathfrak{p}}^{+}}\|B\|+\frac{h\left(\hat{\sigma}_{\mathfrak{p}}\right)^{+}}{1-d_{p}^{+}}<1$, yields $T$ is contractive on set $\mathcal{B}$. Thus, $T$ possesses a unique fixed point $\psi^{*} \in \mathcal{B}$ such that $T \psi^{*}=\psi^{*}$, it follows from (3.1) that $x^{*}=\mathcal{A}^{-1} \psi^{*} \in \mathcal{B}$ is the unique solution of (2.3).

\section{Stability of solution of system (2.3)}

Theorem 4.1. Under the conditions of Theorem 3.1 and $\mathrm{F}(0)=\mathrm{G}(0)=0$, the system (2.3) has a unique solution which is asymptotically stable in mean square if there exist positive definite matrices $\mathrm{P}_{\mathrm{i}}, \mathrm{i} \in \mathrm{S}, \mathrm{Q}$ and positive definite diagonal matrices $v_{1 i}$ and $\omega_{1 i}, i \in S$ such that the following LMI holds:

$$
\Phi_{i}=\left(\begin{array}{cc}
\hat{\Phi}_{i} & M_{i}^{\top} \hat{Q} \\
\hat{Q} M_{i} & -\hat{Q}
\end{array}\right)<0,
$$

where $\hat{Q}=2\left(1+d_{p}^{+}\right) P(i) C(i)$,

$$
M_{i}=[0, B(i), 0, C(i)], \quad \hat{\Phi}_{i}=\left(\begin{array}{cccc}
\Xi_{11 i} & \Xi_{12 i} & \omega_{1 i} \omega_{2} & P_{i} B(i) \\
* & -v_{1 i} & 0 & 0 \\
* & * & \Xi_{33 i} & 0 \\
* & * & * & -Q
\end{array}\right),
$$

with $\hat{P}_{i}=\sum_{j=1}^{n_{0}} \pi_{i j} P_{j}, \Xi_{11 i}=\hat{P}_{i}-v_{1 i} v_{1}-\omega_{1 i} \omega_{1}, \Xi_{12 i}=\hat{P}_{i}+v_{1 i} v_{1}, \Xi_{33 i}=[1+\bar{\pi}(\bar{\tau}-\underline{\tau})] Q-\omega_{1 i}$.

Proof. Construct the following Lyapunov-Krasovskii functional for the system (2.3):

$$
V\left(x_{t}, t, r(t)\right)=\sum_{k=1}^{3} V_{k}\left(x_{t}, t, r(t)\right),
$$

where

$$
\begin{aligned}
& \mathrm{V}_{1}\left(x_{\mathrm{t}}, \mathrm{t}, \mathrm{r}(\mathrm{t})\right)=(\mathcal{A x}(\mathrm{t}))^{\top} \mathrm{P}_{\mathrm{r}(\mathrm{t})} \mathcal{A} x(\mathrm{t}) \\
& \mathrm{V}_{2}\left(x_{\mathrm{t}}, \mathrm{t}, \mathrm{r}(\mathrm{t})\right)=\int_{\mathrm{t}-\tau(\mathrm{r}(\mathrm{t}))}^{\mathrm{t}} \mathrm{G}^{\top}(x(\mathrm{~s})) \mathrm{QG}(x(\mathrm{~s})) \mathrm{ds}, \quad \mathrm{V}_{3}\left(x_{\mathrm{t}}, \mathrm{t}, \mathrm{r}(\mathrm{t})\right)=\bar{\pi} \int_{\underline{\tau}}^{\bar{\tau}} \int_{t-s}^{\mathrm{t}} \mathrm{G}^{\top}(x(\theta)) \mathrm{QG}(x(\theta)) \mathrm{d} \theta \mathrm{ds} .
\end{aligned}
$$


According to the Itô's rule, the weak infinitesimal operator $\mathcal{L}(\cdot)$ of the process $\{x(t), t \geqslant 0, r(t)\}$ for system (2.3) at the point $\{x(t), t \geqslant 0, i\}$ are given by

$$
\begin{aligned}
\mathcal{L} V_{1}\left(x_{t}, t, i\right)= & 2(\mathcal{A x}(t))^{\top} P_{i}[-C(i) x(t)+A(i) F(x(t))+B(i) G(x(t-\tau(i)))]+(\mathcal{A} x(t))^{\top} \hat{P}_{i} \mathcal{A} x(t), \\
\mathcal{L} V_{2}\left(x_{t}, t, i\right)= & G^{\top}(x(t)) Q G(x(t))-G^{\top}(x(t-\tau(i))) Q G(x(t-\tau(i))) \\
& +\sum_{j \neq i}^{n_{0}} \pi_{i j} \int_{t-\tau(j)}^{t} G^{\top}(x(s)) Q G(x(s)) d s+\pi_{i i} \int_{t-\tau(i)}^{t} G^{\top}(x(s)) Q G(x(s)) d s \\
\leqslant & G^{\top}(x(t)) Q G(x(t))-G^{\top}(x(t-\tau(i))) Q G(x(t-\tau(i))) \\
& +\sum_{j \neq i}^{n_{0}} \pi_{i j} \int_{t-\tau(j)}^{t} G^{\top}(x(s)) Q G(x(s)) d s+\pi_{i i} \int_{t-\tau}^{t} G^{\top}(x(s)) Q G(x(s)) d s \\
= & G^{\top}(x(t)) Q G(x(t))-G^{\top}(x(t-\tau(i))) Q G(x(t-\tau(i))) \\
& -\pi_{i i} \int_{t-\tau(j)}^{t} G^{\top}(x(s)) Q G(x(s)) d s+\pi_{i i} \int_{t-\underline{\tau}}^{t} G^{\top}(x(s)) Q G(x(s)) d s \\
\leqslant & G^{\top}(x(t)) Q G(x(t))-G^{\top}(x(t-\tau(i))) Q G(x(t-\tau(i)))+\bar{\pi} \int_{t-\bar{\tau}}^{t-\underline{\tau}} G^{\top}(x(s)) Q G(x(s)) d s, \\
\mathcal{L} V_{3}\left(x_{t}, t, i\right)= & \bar{\pi}(\bar{\tau}-\underline{\tau}) G^{\top}(x(t)) Q G(x(t))-\bar{\pi} \int_{t-\tau}^{t} G^{\top}(x(s)) Q G(x(s)) d s .
\end{aligned}
$$

Substituting (4.4)-(4.6) into (4.3), we obtain

$$
\begin{aligned}
\mathcal{L V}\left(x_{t}, t, i\right)= & 2(\mathcal{A x}(t))^{\top} P_{i}[-\mathrm{C}(i) x(t)+A(i) F(x(t))+B(i) G(x(t-\tau(i)))]+(\mathcal{A} x(t))^{\top} \hat{P}_{i} \mathcal{A} x(t) \\
& +G^{\top}(x(t)) Q G(x(t))-G^{\top}(x(t-\tau(i))) Q G(x(t-\tau(i)))+\bar{\pi}(\bar{\tau}-\underline{\tau}) G^{\top}(x(t)) Q G(x(t)) .
\end{aligned}
$$

According to Lemma 2.6, we have

$$
\begin{array}{r}
(\mathcal{A x}(\mathrm{t}))^{\top} v_{1 i} v_{1} \mathcal{A} x(\mathrm{t})-2(\mathcal{A} x(\mathrm{t}))^{\top} v_{1 i} v_{2} \mathrm{~F}(x(\mathrm{t}))+\mathrm{F}^{\top}(x(\mathrm{t})) v_{1 i} \mathrm{~F}(x(\mathrm{t})) \leqslant 0, \\
(\mathcal{A x}(\mathrm{t}))^{\top} \omega_{1 i} \omega_{1} \mathcal{A} x(\mathrm{t})-2(\mathcal{A} x(\mathrm{t}))^{\top} \omega_{1 i} \omega_{2} \mathrm{G}(x(\mathrm{t}))+\mathrm{G}^{\top}(x(\mathrm{t})) \omega_{1 i} \mathrm{G}(x(\mathrm{t})) \leqslant 0 .
\end{array}
$$

From (4.7)-(4.9), we have

$$
\begin{aligned}
\mathcal{L} V\left(x_{t}, t, i\right)= & 2(\mathcal{A} x(t))^{\top} P_{i}[A(i) F(x(t))+B(i) G(x(t-\tau(i)))]+(\mathcal{A} x(t))^{\top} \hat{P}_{i} \mathcal{A} x(t) \\
& +G^{\top}(x(t)) Q G(x(t))-G^{\top}(x(t-\tau(i))) Q G(x(t-\tau(i)))+\bar{\pi}(\bar{\tau}-\tau) G^{\top}(x(t)) Q G(x(t)) \\
& -(\mathcal{A x}(t))^{\top} v_{1 i} v_{1} \mathcal{A} x(t)+2(\mathcal{A} x(t))^{\top} v_{1 i} v_{2} F(x(t))-F^{\top}(x(t)) v_{1 i} F(x(t)) \\
& -(\mathcal{A} x(t))^{\top} \omega_{1 i} \omega_{1} \mathcal{A} x(t)+2(\mathcal{A} x(t))^{\top} \omega_{1 i} \omega_{2} G(x(t))-G^{\top}(x(t)) \omega_{1 i} G(x(t)) \\
& +2\left(1+d_{p}^{+}\right) x^{\top}(t) P(i) C(i) x(t) \\
\leqslant & \xi_{i}^{\top}\left(x_{t}\right) \hat{\Psi}_{i} \xi_{i}\left(x_{t}\right)+\xi_{i}^{\top}\left(x_{t}\right) M_{i}^{\top} \hat{Q} M_{i} \xi_{i}\left(x_{t}\right)
\end{aligned}
$$

where

$$
\xi_{i}\left(x_{t}\right)=\left[(\mathcal{A} x(t))^{\top}, F^{\top}(x(t)), G^{\top}(x(t)), G^{\top}(x(t-\tau(i)))\right]^{\top},
$$

$\hat{\Psi}_{i}$ and $M_{i}$ are defined by (4.2). From Lemma 2.5, inequality (4.1) is equivalent to

$$
\hat{\Psi}_{i}+M_{i}^{\top} \hat{Q} M_{i}<0 .
$$

Let $\lambda_{0}=\max _{i \in S}\left\{\lambda_{\max }\left(\hat{\Psi}_{i}+M_{i}^{\top} \hat{Q} M_{i}\right)\right\}$, then $\lambda_{0}<0$. In view of (4.10), we have

$$
\mathcal{L} V\left(x_{t}, t, i\right) \leqslant \lambda_{0} \xi_{i}^{\top}\left(x_{t}\right) \xi_{i}\left(x_{t}\right) \leqslant \lambda_{0}|\mathcal{A} x(t)|^{2} .
$$

By Lemma 2.4, we have 


$$
|x(t)|=\left|\mathcal{A}^{-1} \mathcal{A} x(t)\right| \leqslant \frac{1}{1-\mathrm{d}_{\mathrm{p}}^{+}}|\mathcal{A} x(\mathrm{t})| \quad \text { and } \quad|\mathcal{A} x(\mathrm{t})|^{2} \geqslant\left(1-\mathrm{d}_{\mathrm{p}}^{+}\right)^{2}|x(\mathrm{t})|^{2},
$$

which together with (4.11) yields

$$
\mathcal{L V}\left(x_{t}, t, i\right) \leqslant \lambda_{0}\left(1-d_{p}^{+}\right)^{2}|x(t)|^{2}:=\lambda_{1}|x(t)|^{2},
$$

where $\lambda_{1}=\lambda_{0}\left(1-d_{p}^{+}\right)^{2}$. Hence, by (4.12) we have

$$
\mathbb{E V}(x(t), t, r(t))=\mathbb{E V}(x(0), 0, r(0))+\mathbb{E} \int_{0}^{t} \mathcal{L} V(x(s), s, r(s)) d s \leqslant \mathbb{E V}(x(0), 0, r(0))+\lambda_{1} \mathbb{E} \int_{0}^{t}|x(s)|^{2} d s
$$

In view of $\lambda_{1}<0$, we know that $\int_{0}^{t} \mathbb{E}|x(s)|^{2} \mathrm{ds}$ is convergent as $t \rightarrow+\infty$. Hence, from Barbalat's Lemma (see [13]), we have $\lim _{t \rightarrow+\infty} \mathbb{E}|x(t)|^{2}=0$.

Next, we give some exponential stability results for the system (2.3).

Theorem 4.2. Under the conditions of Theorem 4.1, there exists a unique solution for system (2.3) which is exponentially stable in mean square.

Proof. Let $\alpha_{0}=\max \left\{\left|l_{p}^{-}\right|,\left|l_{p}^{+}\right|,\left|\sigma_{p}^{-}\right|,\left|\sigma_{p}^{+}\right|, p=1,2 \cdots, n\right\}$. Using Assumption 2.3 and $F(x)=G(x)=0$, we have

$$
|F(x)|^{2} \leqslant \alpha_{0}^{2}|x|^{2}, \quad|G(x)|^{2} \leqslant \alpha_{0}^{2}|x|^{2} .
$$

From $\hat{\Psi}_{i}+M_{i}^{\top} \hat{Q} M_{i}<0$, there exists a sufficient small positive number $\varepsilon_{0}$ such that

$$
\hat{\Psi}_{i}+M_{i}^{\top} \hat{Q} M_{i}+\varepsilon_{0} I<0,
$$

where I is the identity matrix. We introduce the following Lyapunov-Krasovskii functional

$$
\hat{V}\left(x_{t}, t, r(t)\right)=V\left(x_{t}, t, r(t)\right)-\frac{\lambda_{1}}{2 \tau} \int_{0}^{\tau} \int_{t-s}^{t}(\mathcal{A x}(\theta))^{\top} \mathcal{A} x(\theta) d \theta d s
$$

where $V\left(x_{t}, t, r(t)\right)$ is defined by (4.3). Then we have

$$
\mathcal{L} \hat{V}\left(x_{t}, t, r(t)\right)=\mathcal{L V}\left(x_{t}, t, r(t)\right)-\frac{\lambda_{1}}{2}(\mathcal{A} x(t))^{\top} \mathcal{A x}(\mathrm{t})+\frac{\lambda_{1}}{2 \tau} \int_{\mathrm{t}-\tau}^{\mathrm{t}}(\mathcal{A} x(s))^{\top} \mathcal{A} x(s) \mathrm{ds} .
$$

In view of $\mathcal{L} V\left(x_{t}, t, r(t)\right)<0$, (4.13), and (4.11), we have

$$
\mathcal{L} \hat{\mathrm{V}}\left(\mathrm{x}_{\mathrm{t}}, \mathrm{t}, \mathrm{r}(\mathrm{t})\right) \leqslant\left(\lambda_{0}-\frac{\lambda_{1}}{2}\right)(\mathcal{A} x(\mathrm{t}))^{\top} \mathcal{A} x(\mathrm{t})+\frac{\lambda_{1}}{2 \tau} \int_{\mathrm{t}-\tau}^{\mathrm{t}}(\mathcal{A} x(\mathrm{~s}))^{\top} \mathcal{A} x(\mathrm{~s}) \mathrm{d} s
$$

Let $\mu>0$. Calculate

$$
\mathcal{L}\left[e^{\mu t} \hat{V}\left(x_{t}, t, r(t)\right)\right]=\mu e^{\mu t} \hat{V}\left(x_{t}, t, r(t)\right)+e^{\mu t} \mathcal{L}\left[\hat{V}\left(x_{t}, t, r(t)\right)\right] .
$$

From the definitions of $\hat{V}\left(x_{t}, t, r(t)\right)$ and $V\left(x_{t}, t, r(t)\right)$, we have

$$
\begin{aligned}
\hat{\mathrm{V}}\left(\mathrm{x}_{\mathrm{t}}, \mathrm{t}, \mathrm{r}(\mathrm{t})\right) \leqslant & \rho_{1}(\mathcal{A} x(\mathrm{t}))^{\top} \mathcal{A} x(\mathrm{t})+\alpha_{0}^{2} \lambda_{\max }(\mathrm{Q}) \int_{\mathrm{t}-\tau}^{\mathrm{t}} x^{\top}(\mathrm{s}) x(\mathrm{~s}) \mathrm{ds} \\
& +\bar{\pi}(\bar{\tau}-\underline{\tau}) \alpha_{0}^{2} \lambda_{\max }(\mathrm{Q}) \int_{\mathrm{t}-\tau}^{\mathrm{t}} x^{\top}(\mathrm{s}) x(\mathrm{~s}) \mathrm{d} s \\
= & \rho_{1}(\mathcal{A} x(\mathrm{t}))^{\top} \mathcal{A} x(\mathrm{t})+\rho_{2} \int_{\mathrm{t}-\tau}^{\mathrm{t}} x^{\top}(\mathrm{s}) x(\mathrm{~s}) \mathrm{d} s \\
\leqslant & \rho_{1}(\mathcal{A} x(\mathrm{t}))^{\top} \mathcal{A} x(\mathrm{t})+\frac{\rho_{2}}{\left(1-\mathrm{d}_{\mathrm{p}}^{+}\right)^{2}} \int_{\mathrm{t}-\tau}^{\mathrm{t}}(\mathcal{A} x(\mathrm{~s}))^{\top} \mathcal{A} x(\mathrm{~s}) \mathrm{d} s
\end{aligned}
$$


where $\rho_{1}=\max _{i \in S}\left\{\lambda_{\max }\left(P_{i}\right)\right\}, \rho_{2}=\alpha_{0}^{2} \lambda_{\max }(Q)(1+\bar{\pi}(\bar{\tau}-\underline{\tau}))$. From (4.13)-(4.16), we have

$$
\mathcal{L}\left[e^{\mu t} \hat{V}\left(x_{t}, t, r(t)\right)\right] \leqslant\left(\mu \rho_{1}+\lambda_{0}-\frac{\lambda_{1}}{2}\right) e^{\mu t}(\mathcal{A x}(t))^{\top} \mathcal{A x}(t)+\left(\frac{\lambda_{1}}{2 \tau}+\frac{\mu \rho_{2}}{\left(1-d_{p}^{+}\right)^{2}}\right) e^{\mu t} \int_{t-\tau}^{t}(\mathcal{A x}(s))^{\top} \mathcal{A} x(s) d s .
$$

Choosing $\mu=\mu_{0}$ and $\lambda_{1}$ leads to

$$
\mu_{0} \rho_{1}+\lambda_{0}-\frac{\lambda_{1}}{2} \leqslant 0, \frac{\lambda_{1}}{2 \tau}+\frac{\mu_{0} \rho_{2}}{\left(1-d_{p}^{+}\right)^{2}} \leqslant 0 .
$$

Thus, we have

$$
\mathcal{L}\left[e^{\mu_{0} t} \hat{V}\left(x_{t}, t, r(t)\right)\right] \leqslant 0
$$

and

$$
\mathbb{E}\left[e^{\mu_{0} t} \hat{V}\left(x_{t}, t, r(t)\right)\right]=\mathbb{E}\left[e^{\mu_{0} t} \hat{V}\left(x_{0}, 0, r(0)\right)\right]+\mathbb{E} \int_{0}^{t} \mathcal{L}\left[e^{\mu_{0} s} \hat{V}\left(x_{s}, s, r(s)\right)\right] d s \leqslant \mathbb{E}\left[e^{\mu_{0} t} \hat{V}\left(x_{0}, 0, r(0)\right)\right]
$$

On the other hand, from the definition of $\hat{V}\left(x_{t}, t, r(t)\right)$, there exits a positive constant $\rho_{3}$ such that

$$
\hat{V}\left(x_{t}, t, r(t)\right) \geqslant \rho_{3}(\mathcal{A} x(t))^{\top} \mathcal{A} x(t) .
$$

From (4.17) and (4.18), we have

$$
\mathbb{E}|\mathcal{A} x(t)|^{2} \leqslant \frac{\hat{V}\left(x_{0}, 0, r(0)\right)}{\rho_{3}} e^{-\mu_{0} t}
$$

From Lemma 2.4 and (4.19), we have

$$
\mathbb{E}|x(t)|^{2} \leqslant \frac{\hat{V}\left(x_{0}, 0, r(0)\right)}{\rho_{3}\left(1-d_{\mathfrak{p}}^{+}\right)^{2}} e^{-\mu_{0} t} .
$$

The proof is completed.

Remark 4.3. In Theorems 4.1 and 4.2, based on LMI approach, some sufficient conditions are obtained to guarantee the asymptotically stable in mean square and exponentially stable in mean square for the system (2.3). We point out that the similar stability results can be obtained as immediate consequences for special cases (e.g., without Markovian jumping parameters or constant delays). On the other hand, our arguments can be easily extended the systems with white noises.

\section{Numerical example}

Example 5.1. In this example, we consider a 2-dimensional neutral-type neural networks with the transition rate matrix being

$$
\left(\begin{array}{cc}
-4 & 1 \\
2 & -3
\end{array}\right)
$$

The other parameters are given as follows:

$$
\begin{aligned}
\gamma_{11} & =6, \gamma_{12}=5, & \tau_{11} & =6, \tau_{12}=7, \\
\mathrm{D}(1) & =\left(\begin{array}{cc}
0.5 & 0 \\
0 & 0.5
\end{array}\right), & \mathrm{D}(2) & =\left(\begin{array}{cc}
0.3 & 0 \\
0 & 0.3
\end{array}\right), \\
\mathrm{C}(1) & =\left(\begin{array}{cc}
0.25 & 0 \\
0 & 0.43
\end{array}\right), & \mathrm{C}(2) & =\left(\begin{array}{cc}
1.3 & 0 \\
0 & 1.5
\end{array}\right), \\
\mathrm{A}(1) & =\left(\begin{array}{cc}
0.3 & 0.3 \\
0.2 & -0.5
\end{array}\right), & \mathrm{A}(2) & =\left(\begin{array}{cc}
0.3 & 0.3 \\
0.1 & -0.5
\end{array}\right),
\end{aligned}
$$




$$
\mathrm{B}(1)=\left(\begin{array}{cc}
0.4 & -0.2 \\
0.1 & 0.3
\end{array}\right), \quad \mathrm{B}(2)=\left(\begin{array}{cc}
0.4 & 0.2 \\
0.6 & 0.3
\end{array}\right) .
$$

The activation functions are given as

$$
\mathrm{F}(\mathrm{x})=\mathrm{G}(\mathrm{x})=\left(\tanh \left(-0.3 \mathrm{x}_{1}\right), \tanh \left(-0.5 \mathrm{x}_{2}\right)\right)^{\top} .
$$

With the above parameters, by using Matlab LMI Toolbox, we solve the LMIs (4.1) and obtain the feasible solution as follows:

$$
\begin{aligned}
P(1) & =\left(\begin{array}{cc}
0.3152 & -0.0013 \\
-0.0013 & 0.3012
\end{array}\right), P(2)=\left(\begin{array}{cc}
0.20102 & -0.0925 \\
-0.0925 & 2.0149
\end{array}\right), Q=\left(\begin{array}{cc}
0.4710 & -0.0122 \\
-0.0122 & 0.4723
\end{array}\right), \\
v_{11} & =0.4652, v_{12}=0.4683, v_{21}=0.7341, \quad v_{22}=0.7302 .
\end{aligned}
$$

Therefore, it follows from Theorem 4.2 that the system (2.3) with given parameters is globally exponentially stable in mean square, which is further verified by the simulation results given in Figs. 1 and 2 under two different sets of initial values.

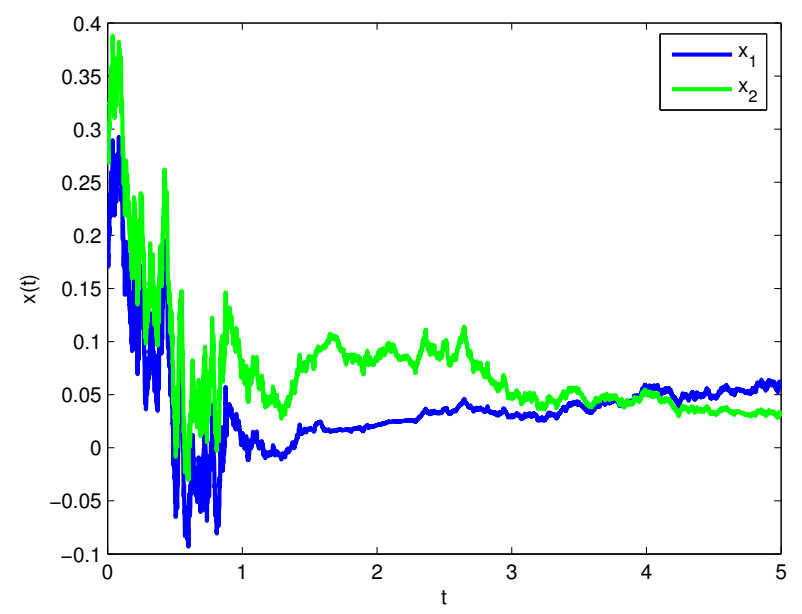

Figure 1: State trajectories of Example 5.1 with initial value $x(0)=(0.2,0.3)$.

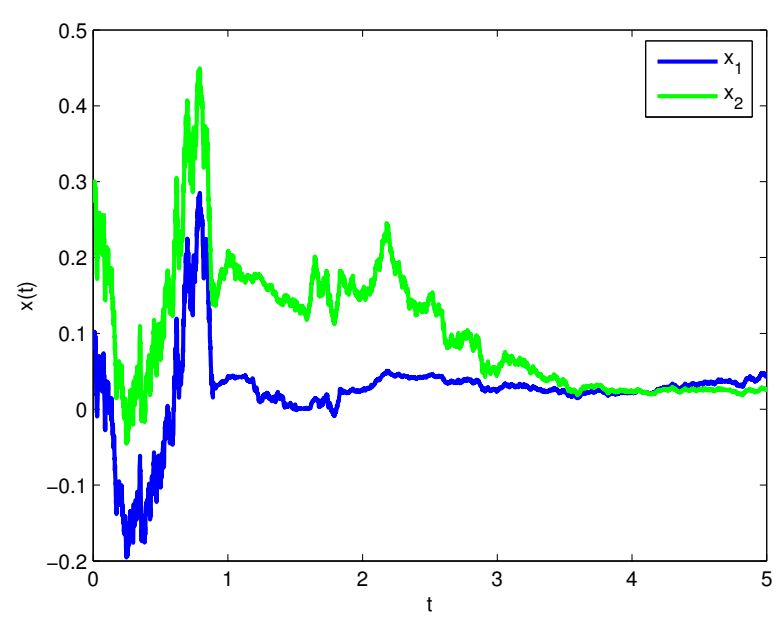

Figure 2: State trajectories of Example 5.1 with initial value $x(0)=(0.4,0.38)$.

Remark 5.2. In our main results, the stability analysis problems are dealt with for a class of neutral-type neural networks with Markovian jumping parameters. A LMI-based sufficient condition is derived for the stability of the neural networks addressed. The exponential stability can be readily checked by the solvability of a set of LMIs, which can be done by resorting to the Matlab LMI toolbox. Note that the LMI (4.1) is delay-dependent, hence less conservative than the traditional delay independent conditions.

\section{Conclusions}

In this paper, we have investigated stability problems for a class of neutral-type neural networks with Markovian parameters and mixed delays. By utilizing novel Lyapunov-Krasovskii functionals, we have established a LMI approach to derive the sufficient conditions guaranteeing the global stability in the mean square of the neural networks. The criteria are expressed in the form of LMIs, which can be solved effectively by using the matlab LMI toolbox. A simulation example has been provided to show the usefulness of the derived LMI-based stability conditions.

\section{Acknowledgment}

This paper is supported by Natural Science Foundation of Jiangsu High Education Institutions of China (Grant No.13KJB110001). 


\section{References}

[1] S. Arik, Global robust stability analysis of neural networks with discrete time delays, Chaos Solitons Fractals, 26 (2005), 1407-1414. 1, 2.2

[2] S. Boyd, L. El Ghaoui, E. Feron, V. Balakrishnan, Linear matrix inequalities in system and control theory, SIAM Studies in Applied Mathematics, Society for Industrial and Applied Mathematics (SIAM), Philadelphia, PA, (1994). 2.5

[3] H.-W. Chen, Z.-M. He, J.-L. Li, Multiplicity of solutions for impulsive differential equation on the half-line via variational methods, Bound. Value Probl., 2016 (2016), 15 pages. 1, 2.2

[4] K. Gu, An integral inequality in the stability problem of time-delay systems, Proceedings of the 39th IEEE Conference on Decision and Control, Sydney Australia, 3 (2000), 2805-2810. 2.7

[5] Z.-J. Gui, W.-G. Ge, X.-S. Yang, Periodic oscillation for a Hopfield neural networks with neutral delays, Phys. Lett. A, 364 (2007), 267-273. 1, 2.2

[6] M. P. Kennedy, L. O. Chua, Neural networks for nonlinear programming, IEEE Trans. Circuits and Systems, 35 (1988), 554-562. 1

[7] Y.-R. Liu, Z.-D. Wang, X.-H. Liu, Exponential synchronization of complex networks with Markovian jump and mixed delays, Phys. Lett. A, 372 (2008), 3986-3998. 1

[8] Y.-R. Liu, Z.-D. Wang, X.-H. Liu, State estimation for discrete-time Markovian jumping neural networks with mixed mode-dependent delays, Phys. Lett. A, 372 (2008), 7147-7155. 2.6

[9] S.-S. Mou, H.-J. Gao, J. Lam, W.-Y. Qiang, A new criterion of delay-dependent asymptotic stability for Hopfield neural networks with time delay, IEEE Trans. Neural Netw., 19 (2008), 532-535. 1, 2.2

[10] J. Pan, X.-Z. Liu, W. -C. Xie, Exponential stability of a class of complex-valued neural networks with time-varying delays, Neurocomputing, 164 (2015), 293-299. 1, 2.2

[11] J. H. Park, C. H. Park, O. M. Kwon, S. M. Lee, A new stability criterion for bidirectional associative memory neural networks of neutral-type, Appl. Math. Comput., 199 (2008), 716-722. 1, 2.1

[12] R. Rakkiyappan, P. Balasubramaniam, J.-D. Cao, Global exponential stability results for neutral-type impulsive neural networks, Nonlinear Anal. Real World Appl., 11 (2010), 122-130. 1, 2.1

[13] J. E. Slotine, W.-P. Li, Applied nonlinear control, Prentice-Hall, Englewood Cliffs, New Jersey, (1991). 4

[14] Z.-D. Wang, Y.-R. Liu, X.-H. Liu, State estimation for jumping recurrent neural networks with discrete and distributed delays, Neural Netw., 22 (2009), 41-48. 1, 2.2

[15] Z.-H. Xia, X.-H. Wang, X.-M. Sun, Q. Wang, A secure and dynamic multi-keyword ranked search scheme over encrypted cloud data, IEEE Trans. Parallel Distrib. Syst., 27 (2015), 340-352. 1

[16] Y. Xu, Z.-M. He, Exponential stability of neutral stochastic delay differential equations with Markovian switching, Appl. Math. Lett., 52 (2016), 64-73. 1, 2.2

[17] K.-W. Yu, C.-H. Lien, Stability criteria for uncertain neutral systems with interval time-varying delays, Chaos Solitons Fractals, 38 (2008), 650-657. 1, 2.1

[18] J. Zhao, D. J. Hill, T. Liu, Global bounded synchronization of general dynamical networks with nonidentical nodes, IEEE Trans. Automat. Controll, 57 (2012), 2656-2662. 1, 2.2 